EPJ Web of Conferences 41, 05009 (2013)

DOI: $10.1051 /$ epjconf/20134105009

(C) Owned by the authors, published by EDP Sciences, 2013

\title{
Dynamics of a photochromic spiropyran under aqueous conditions
}

\author{
J. Kohl-Landgraf ${ }^{1}$, M. Braun ${ }^{1}$, C. Özçoban ${ }^{2}$, D. P. N. Goncalves ${ }^{2}$, A. Heckel ${ }^{2}$ and J. Wachtveitl ${ }^{1}$ \\ ${ }^{1}$ Institute of Physical and Theoretical Chemistry Goethe-Universität, 60438 Frankfurt/Main, \\ Germany \\ ${ }^{2}$ Institute for Organic Chemistry and Chemical Biology, Buchmann Institute for Molecular Life \\ Sciences, Cluster of Excellence Macromolecular Complexes, Goethe University Frankfurt, 60438 \\ Frankfurt/Main, Germany
}

\begin{abstract}
The dynamics of a water soluble spiropyran is investigated by means of femtosecond transient absorption spectroscopy in the visible and infrared spectral range revealing an ultrafast reversible switching behavior under aqueous conditions with a high fatigue resistance.
\end{abstract}

\section{Introduction}

Spiropyrans are photochromic molecules that undergo a reversible structural change upon absorbing a photon of appropriate wavelength [1]. Irradiating spiropyran with UV light leads to the cleavage of the bond between the spiro carbon atom and the oxygen of the chromene moiety and a subsequent isomerization around the three central $\mathrm{C}-\mathrm{C}$ bonds leading to a planar structure called merocyanine. This planar form allows the initially localized $\pi$-systems to stretch across the entire structure resulting in an absorption band in the visible spectral range [2,3]. The photo-dynamics of various derivatives of $1^{\prime}, 3^{\prime}, 3^{\prime}$-trimethylspiro[-2H-1-benzopyran-2,2-indoline] (BIPS) have been studied intensively in different solvents revealing that the opening process leading to the merocyanine form occurs on a picosecond timescale [4]. Although the application of spiro compounds especially in biological systems is promising [5,6], there are essentially no studies on the photochemical behavior of these compounds under aqueous conditions.

We investigated a spiropyran with an enhanced water solubility (Figure 1) due to a pyridinium structure in the right part of the molecule that could be switched between the closed spiropyran and the open merocyanine form numerous times and also appears to be stable in solution for several weeks in contrast to spiro compounds used in earlier experiments where a decomposition took place within hours $[9,10]$. Our time-resolved measurements reveal that the opening reaction takes place within $1.6 \mathrm{ps}$ via the S1 state, whereas the closing reaction is accomplished within $25 \mathrm{ps}$ after relaxation of the excited state via the merocyanine ground state. This is in accordance with the predictions made by Sheng et al. [8]. Its high stability and ultrafast switching in the low picosecond range under aqueous conditions make this compound a versatile tool especially for biological applications [10].

This is an Open Access article distributed under the terms of the Creative Commons Attribution License 2.0, which permits unrestricted use, distribution, and reproduction in any medium, provided the original work is properly cited. 


\section{Materials and Methods}

A Clark CPA 2001 served as source for the fs laser pulses with a central wavelength of $775 \mathrm{~nm}$ and an average output power of $800 \mathrm{~mW}$. For the experiments in the visible range the sample was diluted in analytical grade water to a concentration of $1 \mathrm{mM}$ and filled into a $1 \mathrm{~mm}$ cuvette that was moved during the measurement and illuminated continuously with filtered light from a halogen lamp to accumulate the closed spiropyran form. For the excitation $350 \mathrm{~nm}$ pulses were used that were produced by using a NOPA process with subsequent sum frequency mixing. The probe light was obtained by focusing a small fraction of the fundamental laser pulses into a $5 \mathrm{~mm}$ thick $\mathrm{CaF}_{2}$ substrate generating a supercontinuum white light. The temporal resolution was $220 \mathrm{fs}$.

In the infrared region we used a setup as described elsewhere to generate the probe light [7]. The sample was diluted in $\mathrm{D}_{2} \mathrm{O}$ to a $20 \mathrm{mM}$ concentration and embedded between two $\mathrm{CaF}_{2}$ windows that were separated by a $50 \mu \mathrm{m}$ teflon spacer. The $520 \mathrm{~nm}$ excitation pulses were produced in a NOPA process. During the measurements the cuvette was illuminated with filtered light from a deuterium lamp (380 $\mathrm{nm} \geq \lambda \geq 320 \mathrm{~nm}$ ) to accumulate the merocyanine form in solution.
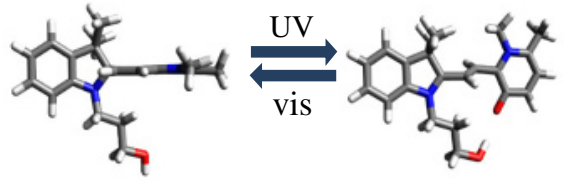

Fig. 1. Structure of the investigated compound: closed spiropyran (left) and its open merocyanine form (right).

\section{Results and Discussion}

The absorption spectrum of the compound is shown in Figure 2. The spectrum of the closed form is dominated by a pronounced peak in the UV range at $350 \mathrm{~nm}$ that can be assigned to a $\mathrm{S} 0 \rightarrow \mathrm{S} 1$ transition in the chromene part of the molecule. After irradiation with UV light a characteristic merocyanine peak appears with an absorption maximum around $520 \mathrm{~nm}$ that originates from the conjugated $\pi$-system of the open form. The molecule is resistant to hydrolysis for more than 1000 hours and it can be switched reversibly for more than 7 times.

To reveal the ultrafast dynamics of the compound fs-transient absorption measurements in the visible and infrared spectral range, shown in Figure 3, were carried out.
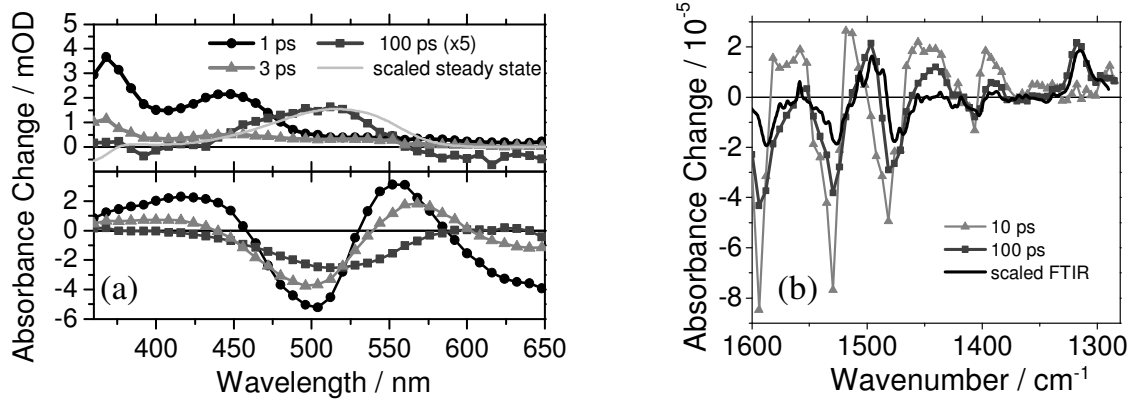

Fig. 3. (a): Transient absorption spectra for certain pump/probe delay times for excitation at $350 \mathrm{~nm}$ (upper part) and $520 \mathrm{~nm}$ (lower part) and probing in the visible range. For comparison a steady state difference spectrum is also plotted. (b) Transient absorption spectra for certain pump/probe delay times in the infrared and scaled FTIR difference spectrum after $520 \mathrm{~nm}$ excitation and probing in the infrared. 
After UV excitation of the closed compound the spectrum is dominated by excited state absorption around $450 \mathrm{~nm}$ and below $400 \mathrm{~nm}$ that both decay with a time constant of $1.6 \mathrm{ps}$ (Figure $3 \mathrm{a}$, upper part). After the relaxation a merocyanine absorbance can be observed that does not change its spectral shape throughout the measurement range. A comparison with the steady state difference spectrum shows that the transient absorption spectrum after 100 ps exhibits a slight blue shift of about $8 \mathrm{~nm}$. The interpretation is that from the $\mathrm{S} 1$ state either, with a 3\% probability, merocyanine is formed or a very efficient relaxation towards the spiropyran ground state takes place. The spectral difference is most likely due to a further thermal isomerization to a different stable isomer or a redistribution of isomers in a time range $>1 \mathrm{~ns}$.

After exciting the open merocyanine with a $520 \mathrm{~nm}$ pulse the spectrum is dominated by an excited state absorption ranging from $350-570 \mathrm{~nm}$ that is intercepted by the ground state bleach centered around 500nm (Figure 3a, lower part). In addition, stimulated emission can be observed from $590 \mathrm{~nm}$ towards longer wavelengths. At longer delay times a decay of the ESA is apparent with a time constant of about 3 ps. Since the product formation could not be observed directly in this spectral region, we also performed vis-pump/IR-probe measurements (Figure 3b). Here one can see that after initial cooling processes a partial repopulation of initially bleached ground state modes takes place indicating a recovery of open merocyanine. On the other hand the formation of characteristic spiropyran bands appears around 1500, 1380, 1320 and weakly at $1560 \mathrm{~cm}^{-1}$ with a rise time of 25 ps. A comparison of the transient absorption spectrum at 100 ps with a scaled FTIR difference spectrum shows very good accordance indicating that the conversion process is finished with the formation of these bands.

A potential application for the compound due to its fast reversible switching, fatigue resistance and good solubility in an aqueous environment is the regulation of biological processes where the molecule could be placed inside a peptide or nucleic acid in such a way that the conformational change of the spiropyran is transferred to its environment and induces a structural change in the respective biological system that leads to reversible switching between an active and an inactive state. This binary behavior can be directly addressed with light of 350 and $520 \mathrm{~nm}$.

Our experiments demonstrate the applicability of water soluble spiropyrans as a trigger in biological systems since it can be switched very fast without decomposition allowing a rapid trigger event for a biological process. Furthermore the rate of hydrolysis is sufficiently low.

\section{References}

1 H. Bouas-Laurent, H. Dürr, Pure Appl. Chem., 73, 639-665 (2001).

2 N. W. Tyer, R. S. Becker, J. Am. Chem. Soc., 92, 1289-1294 (1970).

3 N. P. Ernsting, Chem. Phys. Lett., 159, 526-531 (1989).

4 M. Rini, J. Dreyer, E. T. J. Nibbering, T. Elsaesser, Chem. Phys. Lett., 374, 13-19 (2003).

5 J. Andersson, S. Li, P. Lincoln, J. Andreasson, J. Am. Chem. Soc., 130, 11836-11837 (2008).

6 D. D. Young, A. Deiters, ChemBioChem, 9, 1225-1228 (2008).

7 P. Hamm, R. A. Kaindl, J. Stenger, Opt. Lett., 25, 1798-1800 (2000).

8 Y. Sheng, J. Leszczynski, A. A. Garcia, R. Rosario, D. Gust, J. Springer, J. Phys. Chem. B, 108, 16233-16243 (2004).

9 T. Stafforst, D. Hilvert, Chem. Commun. (2009), 287-288.

10 J. Kohl-Landgraf, M. Braun, C. Özçoban, D. P. N. Gonçalves, A. Heckel, J. Wachtveitl, J. Am. Chem. Soc., (submitted) 